\title{
A IMPORTÂNCIA DO AUTOCUIDADO NA HANSENÍASE: UMA REVISÃO INTEGRATIVA
}

Ilga Milla Chaves Silva1, Fabíola Eloise Rodrigues Dias², Randerson José de Araujo Sousa1, Ana Gabriela de Sousa Costa ${ }^{1}$, Ligia Amaral Filgueiras ${ }^{3}$, Yane Santos Almeida ${ }^{4}$

${ }^{1}$ Graduando do Curso de Medicina, Centro de Ciências Biológicas e da Saúde, Universidade do Estado do Pará, Santarém, PA, Brasil.

${ }^{2}$ Graduanda do Curso de Bacharelado em Fisioterapia, Centro de Ciências Biológicas e da Saúde, Universidade do Estado do Pará, Santarém, PA, Brasil.

${ }^{3}$ Doutora em Antropologia. Prof ${ }^{a}$ no Centro de Ciências Biológicas e da Saúde, Universidade do Estado do Pará, Santarém, Pará, Brasil.

${ }^{4}$ Mestra em Biotecnologia. Profa no Centro de Ciências Biológicas e da Saúde, Universidade do Estado do Pará, Santarém, Pará, Brasil.

Email para correspondência: i.mylla.cs@gmail.com

\section{Resumo}

Objetivo: reconhecer qual a importância do autocuidado na hanseníase e como as ações educativas possibilitam compreender os empasses e o processamento da doença. Metodologia: revisão integrativa, feita no período de 2010 a 2019, onde se utilizou as bases de dados: BVS, SciELO e PubMed, e descritores em português, inglês e espanhol, junto aos critérios de inclusão e exclusão. Os artigos selecionados foram organizados por título, autores, ano, local, amostra, tipo de estudo e resultados apresentados/conclusões e, por fim, foram discutidos. Resultados: observaram-se 120 artigos, porém apenas nove se encaixaram na temática. Os estudos apontaram que hansenianos enfrentam desafios devido às limitações físicas, e sofrem danos psicológicos dado o abandono, preconceito e estigma social. Nesse sentido, o incentivo ao autocuidado associado ao estímulo à inclusão na sociedade, por meio dos grupos de apoio, foi considerado eficaz na recuperação e bem-estar ao longo do tratamento. Conclusão: o maior desafio do autocuidado está no compartilhamento de informações que é feito em grande parte pela educação em saúde, porém é falho, o que impacta na não adesão ao tratamento, sobretudo, de pacientes menos esclarecidos e proporciona prejuízo à imagem corporal, a permanência do preconceito e da exclusão social.

Palavras-chave: Educação em saúde, Preconceito, Imagem corporal, Estigma social.

\begin{abstract}
Objective: to recognize the importance of self-care in leprosy and how educational actions make it possible to understand the impasses and the processing of the disease. Methodology: integrative review, carried out from 2010 to 2019, where the databases: VHL, SciELO and PubMed, and descriptors in Portuguese, English and
\end{abstract}




\begin{abstract}
Spanish were used, along with the inclusion and exclusion criteria. The selected articles were organized by title, authors, year, location, sample, type of study and results presented / conclusions and, finally, were discussed. Results: 120 articles were observed, but only nine fit the theme. Studies have shown that leprosy people face challenges due to physical limitations, and suffer psychological damage due to abandonment, prejudice and social stigma. In this sense, encouraging self-care associated with encouraging inclusion in society, through support groups, was considered effective in recovery and well-being throughout treatment. Conclusion: the biggest challenge of self-care is in the sharing of information that is done in large part by health education, but it is flawed, which impacts on non-adherence to treatment, especially for less enlightened patients and provides damage to body image, permanence of prejudice and social exclusion.
\end{abstract}

Keywords: Health Education, Prejudice, Body Image, Social Stigma.

\title{
1 Introdução
}

A hanseníase é uma doença milenar e que apesar dos avanços científicos continua sendo um problema de saúde pública no Brasil pelo seu poder de causar incapacidade física, social e econômica (GALAN et al., 2016; RIBEIRO; SILVA; OLIVEIRA, 2018). É causada pelo Mycobacterium leprae e é caracterizada por ser uma doença infectocontagiosa, mas de baixa patogenicidade, ou seja, pode infectar inúmeras pessoas, porém somente algumas poderão evoluir com sintomas (SILVA; LOPES; COSTA, 2019). De acordo com a Organização Mundial da Saúde, no ano de 2015 foram detectados 210.758 novos casos em todo o mundo, sendo o Brasil o segundo país que apresentou as maiores notificações, com 26.395 casos (WORLD HEALTH ORGANIZATION, 2016).

Nesse cenário epidemiológico, o Brasil apresenta uma distribuição espacial heterogênea da doença, que é influenciada por variáveis socioeconômicas, demográficas e de políticas públicas, nas quais se destacam as condições de vida desfavoráveis das populações humanas e as dificuldades de acesso aos serviços de saúde, fatores que elevam a concentração de casos novos nas regiões Norte, Nordeste e Centro-Oeste, territórios com alta carga da doença (GONÇALVES et al., 2018; BRASIL, 2020; REIS et al., 2019).

Essa infecção afeta principalmente a pele e os nervos periféricos com capacidade de ocasionar lesões neurais, conferindo à doença um alto poder incapacitante, principal responsável pelo estigma e discriminação às pessoas acometidas por essa patologia e que atinge diretamente a estabilidade emocional, social e produtiva do indivíduo (BRASIL, 2020; LIMA et al., 2018). O 
Brasil, por meio do Sistema Único de Saúde (SUS) disponibiliza o tratamento e o acompanhamento da doença em unidades básicas de saúde e em unidades de referência para tratamento de hanseníase (BRASIL, 2020).

No entanto, a dificuldade de acesso à rede de serviços de saúde pelas populações mais vulneráveis, assim como a forma fragmentada, mecânica e linear desses serviços condiciona o indivíduo a repetir padrões antigos, tornando-o dependente dos profissionais de saúde (SOUZA et al., 2014). Nesse sentido, como forma de prevenir o avanço de incapacidades já instaladas e de futuras incapacidades e deformidades, o Ministério da Saúde, por meio do Programa de Controle da Hanseníase, propõe ações educativas em autocuidado (BATISTA; VIEIRA; DE PAULA, 2014).

As práticas de autocuidado, por sua vez, visam à superação das limitações do hanseniano, proporcionando a troca de experiências, o desenvolvimento das potencialidades e favorecendo a sua autonomia para a melhoria, sobretudo, da qualidade de vida (BRASIL, 2010). Dessa forma, este estudo objetivou realizar uma revisão da literatura, acerca de reconhecer qual é a importância do autocuidado na hanseníase e como as ações educativas possibilitam compreender os empasses e o processamento da doença nos indivíduos.

\section{Metodologia}

Este estudo consistiu em uma revisão da literatura do tipo integrativa, as quais são um método de pesquisa que buscam realizar uma análise de determinado tema outrora investigado e que já possui artigos publicados. Em síntese, a revisão integrativa possibilita o surgimento de novos conhecimentos científicos a partir da avaliação e resumo de pesquisas já relatadas na literatura (SANTOS; ARAÚJO; OLIVEIRA, 2020).

$\mathrm{Na}$ construção desse artigo, adotou-se o período de 2010 a 2019 e seguiu-se as etapas propostas por Souza et al. (2010) e Soares et al. (2019) em que, primeiramente, estabeleceu-se a pergunta que norteou a investigação. Em seguida, para a realização do levantamento bibliográfico, utilizaram-se as respectivas bases de dados: Biblioteca Virtual em Saúde (BVS), Scientific Electronic Library Online (SciELO) e PubMed, com os seguintes descritores em 
português e suas combinações em inglês e espanhol: "hanseníase" (leprosy/ lepra); "autocuidado" (self care/ autocuidado); "qualidade de vida" (quality of life/ calidad de vida); "educação em saúde" (health education/ educación en salud); "saúde pública" (public health/ salud pública).

Ao passo, para a seleção e análise crítica dos artigos catalogados, seguiram-se as respectivas etapas: 1) leu-se o título e o resumo brevemente; 2) consideraram-se os seguintes critérios de inclusão: artigos publicados em língua inglesa, portuguesa e/ou espanhola, estudos científicos disponíveis integralmente e os produzidos com a realidade brasileira, bem como, os seguintes critérios de exclusão: artigos que tangenciavam a temática proposta, teses, dissertações, trabalhos de conclusão de curso, artigos de revisão, perfis epidemiológicos, estudos clínicos e randomizados; 3) quando adequados, realizou-se a leitura na íntegra e a retirada de informações para a revisão.

As informações que constituíram os resultados foram organizadas em um quadro construído com o auxílio do software Microsoft Exce/®2010, o qual foi estruturado em: título do artigo, autores, ano de publicação, local realizado, amostra, tipo de estudo, resultados apresentados e conclusões. Posteriormente, os resultados evidenciados foram discutidos e as lacunas, inferências e vieses pontuados, quando pertinentes. Por fim, dissertou-se de modo claro e completo possibilitando a análise crítica do leitor, sendo todas as etapas, rigorosamente, cumpridas e as condições éticas respeitadas.

\section{$3 \quad$ Resultados e Discussão}

No período delimitado para a realização do levantamento bibliográfico, foram catalogados 120 artigos nas plataformas selecionadas seguindo os critérios de inclusão e exclusão estabelecidos. Destes, apenas 9 se adequaram à temática proposta nesta revisão e os outros 111 foram desconsiderados. Os anos em que se encontrou maior número de artigos, independentemente do objetivo, foram 2019, com 23, seguido do ano 2018, com 15 estudos.

$\mathrm{O}$ ano em que mais se incluíram artigos seguindo a ideia objetivada foi 2018, sendo 3 no período. Nos anos de 2011, 2012, 2013, 2015 e 2017, não foi considerado nenhum artigo. Identificaram-se no método que quatro artigos desenvolveram estudos descritivo/qualitativo, um documental, um 
observacional, um transversal descritivo e outro transversal com abordagem quanti-qualitativa. Todos os estudos incluídos estão sintetizados no Quadro 1.

A hanseníase é uma infecção cercada de muitos estigmas e descaracterizá-los é fundamental para que os pacientes se sintam mais acolhidos no âmbito social e da assistência em saúde, e essa desconstrução começa quando o indivíduo acometido reconhece sua condição diante da infeção, ou seja, a autocompreensão acerca do seu corpo e a ressignificação da sua autoimagem colaboram para uma maior adesão ao tratamento diante do expressivo desinteresse pela terapêutica (BATISTA; VIEIRA; DE PAULA, 2014).

No entanto, muitos pacientes, por receio de sofrerem represálias nas múltiplas esferas sociais, acabam ocultando a sua condição de hanseniano e isso corrobora para que eles escondam, também, a patologia de si mesmos, sendo esse um dos fatores para que haja a rejeição dos cuidados requeridos ou a não incorporação do tratamento de forma aceitável por parte do paciente, além do mais, outra análise pertinente para as dificuldades da adesão ao autocuidado recaem na questão de que as práticas são pautadas em uma abordagem exclusivamente tecnicista, ou seja, intervenções que para 0 hanseniano são extremamente dificultosas (GALAN et al., 2016).

Quadro 1 - Síntese dos artigos científicos incluídos, acerca do autocuidado na hanseníase, entre os anos de 2010 a 2019, Brasil.

\begin{tabular}{|c|c|c|c|}
\hline $\begin{array}{c}\text { Título/autores } \\
\text { Ano/local }\end{array}$ & Amostra & $\begin{array}{l}\text { Tipo de } \\
\text { estudo }\end{array}$ & $\begin{array}{c}\text { Resultados apresentados/ } \\
\text { Conclusões }\end{array}$ \\
\hline $\begin{array}{lr}\text { Prevenção } & \text { de } \\
\text { incapacidade } & \text { na } \\
\text { hanseníase } & \text { com } \\
\text { apoio em } & \text { um } \\
\text { manual } & \text { de } \\
\text { autocuidado para } \\
\text { pacientes } \\
\text { Rodini et al., } 2010 \\
\text { São Paulo }\end{array}$ & $\begin{array}{c}26 \\
\text { pacientes }\end{array}$ & $\begin{array}{c}\text { Observacional } \\
\text { e abordagem } \\
\text { qualitativa }\end{array}$ & $\begin{array}{l}\text { Os pacientes receberam } \\
\text { orientações durante } \\
\text { tratamento, a fim de } \\
\text { conhecerem sobre a } \\
\text { doença, aderirem ao } \\
\text { tratamento e, dessa forma, } \\
\text { prevenir as incapacidades. } \\
\text { Com isso, notou-se melhoria } \\
\text { na função muscular das } \\
\text { mãos, pés e do }\end{array}$ \\
\hline
\end{tabular}




\begin{tabular}{|c|c|c|c|}
\hline & & & $\begin{array}{l}\text { ressecamento da pele, } \\
\text { supostamente ligadas ao } \\
\text { manual, que dispõe acerca } \\
\text { do autocuidado. }\end{array}$ \\
\hline $\begin{array}{l}\text { Conhecimento } \\
\text { sobre prevenção } \\
\text { de incapacidades } \\
\text { em um grupo de } \\
\text { autocuidado em } \\
\text { hanseníase } \\
\\
\text { Pinheiro et al., } \\
2014 \\
\text { Rio Grande do } \\
\text { Norte }\end{array}$ & $\begin{array}{c}16 \\
\text { pacientes }\end{array}$ & $\begin{array}{l}\text { Descritivo e } \\
\text { abordagem } \\
\text { qualitativa }\end{array}$ & $\begin{array}{l}\text { Realizou-se duas reuniões e } \\
\text { uma oficina instrutivas e } \\
\text { após isso os participantes } \\
\text { interagiram sobre o que } \\
\text { aprenderam. Foi constatado } \\
\text { que a participação nos } \\
\text { grupos é essencial para } \\
\text { adquirirem conhecimentos } \\
\text { sobre o autocuidado, sendo } \\
\text { que o enfermeiro possui } \\
\text { papel crucial na formulação } \\
\text { dessas atividades. Destaca- } \\
\text { se que a reabilitação } \\
\text { socioeconômica realizada } \\
\text { ajuda os pacientes a } \\
\text { reinserirem-se na sociedade } \\
\text { e buscarem autonomia } \\
\text { financeira. }\end{array}$ \\
\hline $\begin{array}{l}\text { Autocuidado na } \\
\text { percepção de } \\
\text { pessoas com } \\
\text { hanseníase sob a } \\
\text { ótica } \\
\text { complexidade } \\
\begin{array}{l}\text { Souza et al., } \\
\text { São Paulo }\end{array} \\
\text { Sal4 }\end{array}$ & $\begin{array}{c}15 \\
\text { pacientes }\end{array}$ & $\begin{array}{c}\text { Descritivo, } \\
\text { exploratório e } \\
\text { qualitativo }\end{array}$ & 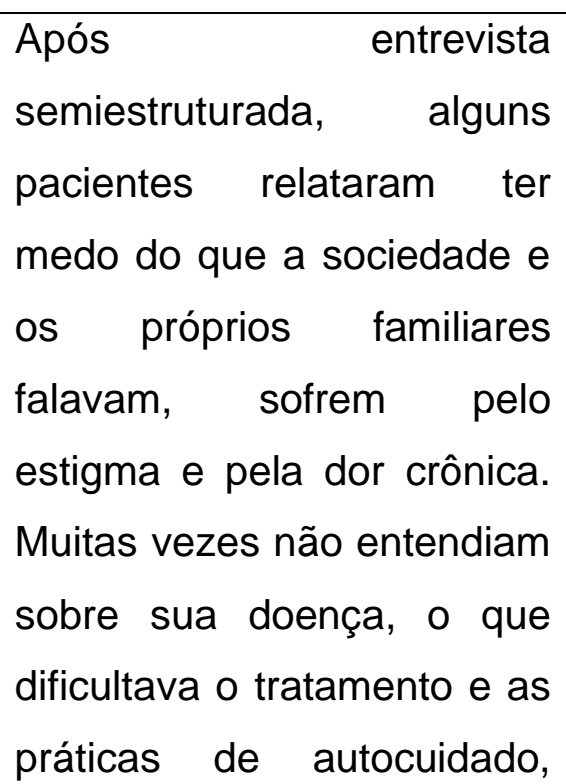 \\
\hline
\end{tabular}




\begin{tabular}{|c|c|c|c|}
\hline & & & 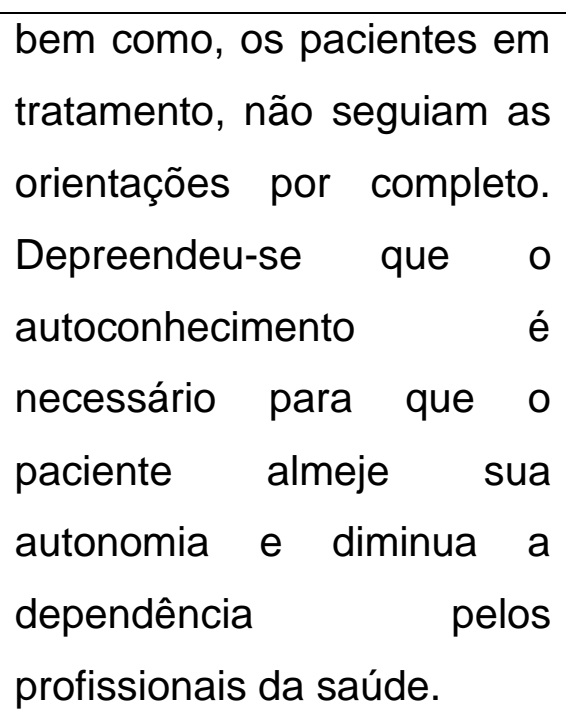 \\
\hline \begin{tabular}{l} 
Avaliação \\
prática do \\
Autocuidado \\
domiciliar \\
hanseníase \\
\\
GALAN et al., \\
2016 \\
\multicolumn{2}{c}{ São Paulo }
\end{tabular} & $\begin{array}{c}11 \\
\text { pacientes }\end{array}$ & $\begin{array}{c}\text { Documental, } \\
\text { observacional, } \\
\text { quantitativo e } \\
\text { descritivo }\end{array}$ & $\begin{array}{l}\text { Analisou-se prontuários e } \\
\text { realizou-se entrevista, após, } \\
\text { constatou-se que a falta de } \\
\text { conhecimento sobre a } \\
\text { doença, o estigma, a } \\
\text { percepção de incapacidade } \\
\text { perante a doença e a } \\
\text { dificuldade no acesso à } \\
\text { saúde são fatores que } \\
\text { interferem no autocuidado. } \\
\text { Por outro lado, o auxílio da } \\
\text { família e a responsabilidade } \\
\text { com a saúde foram fatores } \\
\text { positivos na adesão ao } \\
\text { autocuidado. }\end{array}$ \\
\hline $\begin{array}{l}\text { The use of } \\
\text { assistive } \\
\text { technology to } \\
\text { promote care of } \\
\text { the self and social } \\
\text { inclusion in } \\
\text { patients with } \\
\text { sequels of leprosy } \\
\text { Maia et al., 2016 } \\
\text { Rio de Janeiro }\end{array}$ & $\begin{array}{c}8 \\
\text { pacientes }\end{array}$ & $\begin{array}{c}\text { Exploratório, } \\
\text { descritivo e } \\
\text { qualitativo }\end{array}$ & $\begin{array}{l}\text { Por meio de entrevista } \\
\text { semiestruturada } \\
\text { observação, o estudo } \\
\text { associou o uso da } \\
\text { tecnologia assistiva, por } \\
\text { meio de talheres e } \\
\text { ferramentas, como forma de } \\
\text { resgatar }\end{array}$ \\
\hline
\end{tabular}




\begin{tabular}{|c|c|c|c|}
\hline & & & $\begin{array}{l}\text { autonomia/autocuidado dos } \\
\text { pacientes e ajudar na } \\
\text { inclusão social. A adaptação } \\
\text { foi positiva e suscitou } \\
\text { sentimentos de felicidade na } \\
\text { maioria dos casos, porém } \\
\text { alguns relataram dor ao } \\
\text { utilizar os equipamentos e } \\
\text { dificuldades quanto ao } \\
\text { manuseio. Outro ponto é a } \\
\text { discriminação social por } \\
\text { usar um instrumento de } \\
\text { autoajuda. }\end{array}$ \\
\hline $\begin{array}{l}\text { Percepção de } \\
\text { pacientes com } \\
\text { hanseníase } \\
\text { acerca dos grupos } \\
\text { de autocuidado } \\
\\
\text { D'Azevedo et al., } \\
2018 \\
\text { Pernambuco }\end{array}$ & $\begin{array}{c}11 \\
\text { pacientes }\end{array}$ & $\begin{array}{l}\text { Descritivo e } \\
\text { qualitativo }\end{array}$ & $\begin{array}{l}\text { A partir de uma entrevista } \\
\text { semiestruturada, a maioria } \\
\text { dos pacientes reconhece os } \\
\text { grupos de autocuidado } \\
\text { como uma forma de difusão } \\
\text { de conhecimentos e } \\
\text { interação social, o que } \\
\text { facilita a adesão ao } \\
\text { tratamento/autocuidado. } \\
\text { Ainda, relataram melhora na } \\
\text { autoestima e superação do } \\
\text { preconceito relacionado à } \\
\text { hanseníase. Por fim, } \\
\text { constatou-se que a } \\
\text { participação nos grupos } \\
\text { diminui as incapacidades } \\
\text { físicas. }\end{array}$ \\
\hline $\begin{array}{l}\text { Práticas } r \\
\text { autocuidado em } \\
\text { hanseníase: face, } \\
\text { mãos e pés }\end{array}$ & $\begin{array}{c}24 \\
\text { pacientes }\end{array}$ & $\begin{array}{l}\text { Descritivo, } \\
\text { exploratório e } \\
\text { qualitativo }\end{array}$ & $\begin{array}{l}\text { Foi realizada entrevista } \\
\text { semiestruturada e os } \\
\text { pacientes relataram receber }\end{array}$ \\
\hline
\end{tabular}




\begin{tabular}{|c|c|c|c|}
\hline $\begin{array}{l}\text { Lima et al., } 2018 \\
\text { Pernambuco }\end{array}$ & & & 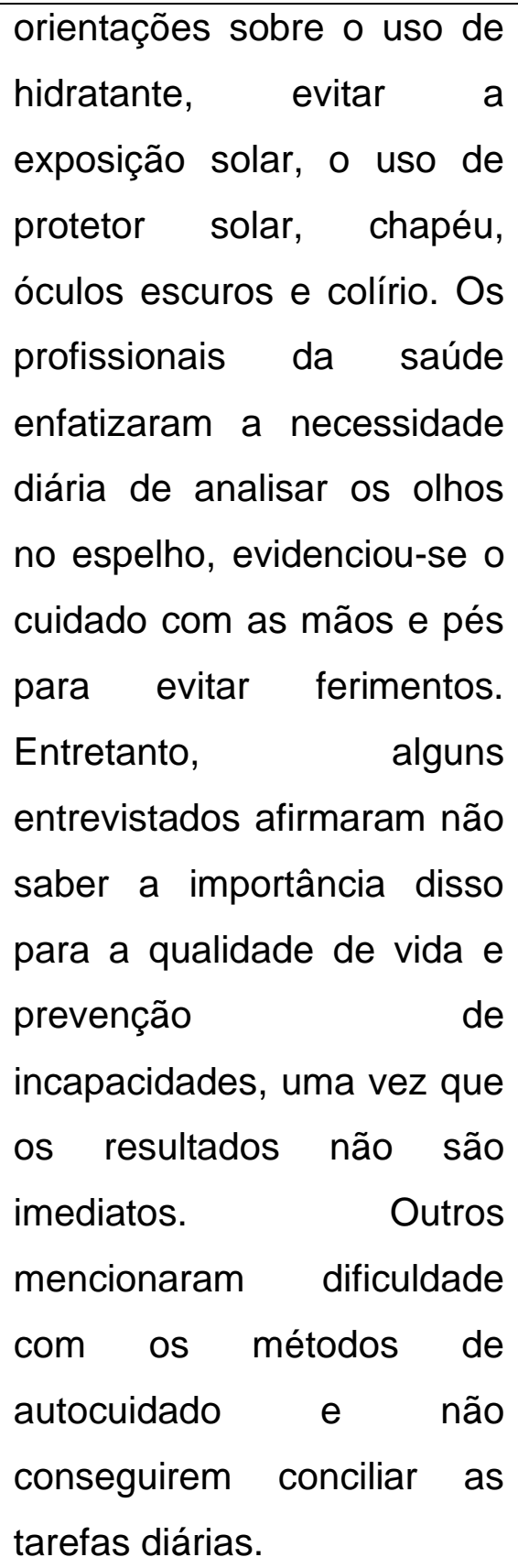 \\
\hline $\begin{array}{l}\text { Acesso rà } \\
\text { orientação quanto } \\
\text { ao autocuidado } \\
\text { por pessoas } \\
\text { diagnosticadas } \\
\text { com hanseníase } \\
\text { em um município } \\
\text { da Zona da Mata } \\
\text { Mineira } \\
\text { Laurindo et al., } \\
2018 \\
\text { Minas Gerais }\end{array}$ & $\begin{array}{c}23 \\
\text { pacientes }\end{array}$ & $\begin{array}{c}\text { Transversal e } \\
\text { descritivo }\end{array}$ & $\begin{array}{l}\text { Após visita domiciliar e } \\
\text { aplicação de questionário, } \\
\text { notou-se que as orientações } \\
\text { dessas unidades de saúde } \\
\text { não são feitas, conforme } \\
\text { preconiza o Ministério da } \\
\text { Saúde (o que pode } \\
\text { contribuir para o aumento } \\
\text { das incapacidades físicas), }\end{array}$ \\
\hline
\end{tabular}




\begin{tabular}{|c|c|c|c|}
\hline & & & $\begin{array}{l}\text { porém há mais informações } \\
\text { sobre olhos e nariz do que } \\
\text { em relação às mãos e pés e } \\
\text { exercício físico. Concluiu-se } \\
\text { que há necessidade de mais } \\
\text { ações educativas } \\
\text { participação dos pacientes. }\end{array}$ \\
\hline $\begin{array}{l}\text { Association } \\
\text { between alcoholic } \\
\text { beverages } \\
\text { consumption } \\
\text { And } \\
\text { sociodemographic } \\
\text { conditions in } \\
\text { people with } \\
\text { leprosy } \\
\text { Dourado et al., } \\
2019 \\
\text { Piauí }\end{array}$ & $\begin{array}{c}290 \\
\text { pacientes }\end{array}$ & $\begin{array}{c}\text { Transversal e } \\
\text { abordagem } \\
\text { quanti- } \\
\text { qualitativa }\end{array}$ & $\begin{array}{l}\text { Utilizou-se formulário de } \\
\text { perguntas e testes durante a } \\
\text { entrevista. } \\
\text { medidas preventivas e } \\
\text { políticas governamentais } \\
\text { acerca desse tema, } \\
\text { porquanto o consumo de } \\
\text { álcool dificulta o autocuidado } \\
\text { e adesão ao tratamento da } \\
\text { hanseníase e atinge } \\
\text { principalmente pessoas com } \\
\text { baixa renda e escolaridade. }\end{array}$ \\
\hline
\end{tabular}

Fonte: elaborado pelos autores, 2020.

Por outro lado, o hanseniano não deve se tornar integralmente dependente das ações do profissional de saúde para que não perca sua independência, mas para isso, percebe-se a relevância da ação de outros setores além das unidades de saúde, já que a participação familiar, por exemplo, é um fator que corrobora para a manutenção da terapia domiciliar, porém só funciona de maneira eficaz quando se esclarece acerca do quadro do paciente para si e para a família, algo nem sempre possível devido aos estigmas da hanseníase (SOUZA et al., 2014; GALAN et al., 2016).

Nesse sentido, a orientação e seu caráter sensibilizador que, embora complexo, permite fazer com que os pacientes compreendam a doença e, assim, possam aderir e seguir corretamente o tratamento recomendado, buscando o atendimento médico quando realmente for crucial e tendo, portanto, autonomia em seus cuidados, afinal, o conhecimento acerca da 
patologia é essencial para a manutenção do autocuidado, uma vez que, é por meio dele que o paciente adquire entendimento dos possíveis desdobramentos da doença e, consequentemente, da relevância de medidas de preservação (SOUZA et al., 2014; LIMA et al., 2018).

Todavia, em face dessa condição de compartilhamento do conhecimento, considerada a ideal dentro da relação profissional da saúde e paciente, que ainda existem falhas evitáveis, mas por vezes compreensíveis, e isso engendra dificuldades no entendimento acerca do porquê usar os métodos de proteção, como óculos escuros, bonés, hidratantes, e outros, ou do porquê seguir corretamente o tratamento (LIMA et al., 2018). Os referidos autores ainda afirmaram que os participantes da pesquisa receberam orientações, mas não as aplicavam cotidianamente, enquanto outros disseram despender tempo em tarefas extras, mas não com o autocuidado.

Em face disso, a dificuldade na divulgação de informações, sem dúvida, é uma barreira a ser superada, pois permeia diferentes realidades no Brasil, como evidenciou um estudo realizado na Zona da Mata Mineira, o qual constatou que os pacientes não detinham orientações necessárias acerca do autocuidado e aos efeitos da doença e dos medicamentos (LAURINDO et al., 2018). Resultado similar foi encontrado em uma pesquisa realizada no município do Rio de Janeiro, a qual identificou que, apesar dos pacientes receberem informações, a impessoalidade dos profissionais ao realizar a educação em saúde tornou o processo algo mais técnico, dificultando a compreensão e a realização do autocuidado pelo doente (SILVA \& PAZ, 2010).

Ao passo, em Imperatriz no Maranhão, correlacionou-se a falta de acompanhamento adequado ao abandono da terapêutica e questões socioeconômicas (SOUSA et al., 2013), realidade que contradiz as determinações do Ministério da Saúde (BRASIL, 2017). Parte disso é novamente devido à dificuldade na troca de um conhecimento com uma linguagem mais acessível entre os profissionais da saúde e pacientes, já que a alta prevalência de baixa escolaridade entre os hansenianos, associada à aplicação de termos especializados pelos profissionais, constitui-se como um empecilho para o entendimento das orientações (ROLIM et al., 2016). 
Esse é um fator que se relaciona, sobretudo, ao despreparo das equipes das unidades, já que, ao longo da formação acadêmica não há conteúdo específico voltado para a abordagem do paciente com hanseníase, devendo o mesmo ser buscado posteriormente e, embora o Ministério da Saúde disponha de orientações essenciais para os cuidados ao hanseniano (BELUCl; BORGATO; GALAN, 2012; BRASIL, 2010), falta à contratação de equipes de apoio especializadas, capacitação profissional e publicidade para que esta doença silenciosa seja percebida pelas ações de saúde pública e, principalmente, desestigmatizada pela sociedade.

Nessa perspectiva, e diante da pluralidade comportamental dos pacientes, é imprescindível o acompanhamento rotineiro com um profissional ou a visita domiciliar para a observação do cotidiano dos hansenianos (LIMA et al., 2018). Contudo, há inúmeras inviabilidades dentro da atenção primária em saúde, como a falta de especialistas, a citar dermatologistas, além disso, quando se trata a hanseníase como uma doença incapacitante e que pode desencadear sequelas, há carência, também, de fisioterapeutas, psicólogos e assistentes sociais, dificultando ainda mais a resolubilidade da doença e que são de grande relevância no contexto da assistência (LAURINDO et al., 2018).

Por tudo isso e vislumbrando a árdua tarefa de disseminação do conhecimento e com ela a laborosa missão de sensibilizar o enfermo, os grupos de autocuidado em hanseníase surgem como uma medida realizada ao nível da atenção básica e que objetiva tornar a terapia um processo mais humanizado, por meio da integração e de trocas de experiências entre hansenianos e profissionais da saúde (BRASIL, 2010). Eles, portanto, mostram-se como estratégias viáveis, pois denotam efetividade quanto à redução de incapacidades e intolerância, além de contribuírem para o aumento à adesão às medidas de autocuidado (D'AZEVEDO et al., 2018).

Diante da efetividade dessa prática coletiva e das inúmeras janelas que ela pode abrir para o hanseniano, um estudo efetivado no estado do Rio Grande do Norte, mostrou que o grupo de autocuidado pesquisado, além da assistência em saúde, promovia atividades educacionais com informações valiosas para melhorar a rotina dos pacientes, prevenir possíveis complicações 
e gerar debates sobre a inserção deles na sociedade e no mercado de trabalho (PINHEIRO et al., 2014).

Em contrapartida, quando se considera as complicações da hanseníase e o impacto do autocuidado sobre elas, as medidas preventivas executadas pelos próprios pacientes não exercem muita influência sobre as incapacidades físicas já estabelecidas, mas são de grande significância para o tratamento de sintomas que possam ser um obstáculo para a qualidade de vida, uma vez que as limitações físicas da patologia exercem repercussões diretas sobre a saúde psicológica do hanseniano, devido à gradual perda de autonomia e rejeição pessoal (RODINI et al., 2010; CORRÊA et al., 2014), porém isso pode ser superado com cuidados alternativos que estimulem o empoderamento e a inclusão social (MAIA et al., 2016).

Assim, além de seguir adequadamente o tratamento, a realização de um diagnóstico precoce é fundamental, pois o bacilo passa a não ser transmissível e os problemas que tangenciam, sobretudo, a incapacidade física são evitáveis, dessa maneira, a análise avaliativa do grau de incapacidade motora configura uma estratégia importante e que é abordada pelo programa de controle da hanseníase, mas como um mecanismo de avaliação da eficiência do programa (LANZA et al., 2012; CASTRO et al., 2009). Nesse contexto, o mais adequado ao paciente seria uma abordagem multiprofissional que possua integração entre si (SOUZA et al., 2014).

Tal integração é, ao passo, estimulada pelo Ministério da Saúde, o qual determina que o paciente deve ter acesso, ao nível da unidade básica, aos cuidados específicos que visem à prevenção de incapacidades sem comprometimento socioeconômico, como a oferta de exercícios físicos e auxílio psicológico, objetivando maior engajamento na terapia domiciliar (BRASIL, 2017). Todavia, esse atendimento integral não é uniforme devido a uma falta de padronização do fluxo de serviços (BARBOSA et al., 2014) e, o Brasil infelizmente ainda possui um descompasso entre a efetividade teórica proposta pelo Programa Nacional de Eliminação da Hanseníase e o sucesso da assistência em saúde para com os pacientes acometidos. 


\section{Conclusão}

A literatura destaca que o conhecimento por parte do hanseniano, acerca da patologia é imprescindível para a adesão ao autocuidado, de modo que, ao praticá-lo, o paciente estará ciente dos benefícios para sua saúde. Entretanto, é válido ressaltar que muitos pacientes, mesmo possuindo informações, não seguem o acompanhamento médico corretamente, logo, é imprescindível identificar criteriosamente os motivos para tal condição.

Assim, como forma de incentivo ao tratamento, os estudos apontaram que o engajamento nos grupos de autocuidado é fundamental para a interação social e troca de experiências, mas que esses encontros só são de fato eficazes, quando a equipe multiprofissional da atenção básica, está capacitada para elaborar ações educativas que gerem impacto na qualidade de vida e transformem a maneira como o hanseniano enfrenta a infecção, o que ainda é um dos grandes empasses na assistência.

Esta revisão denotou, portanto, que embora a autonomia do paciente seja a chave do autocuidado, muitos desafios barram a concretização dessa prática, sobretudo, quando se pauta os âmbitos: sociocultural, socioeconômico e de assistência à saúde, os quais refletem inúmeras problemáticas que atingem diretamente a forma como a hanseníase se processa nos indivíduos, na família e nos profissionais de saúde.

Por isso, estudos direcionados e mais detalhados, com novas abordagens metodológicas a respeito de como os aspectos sociais, econômicos e de assistência à saúde impactam no processamento da doença precisam ser realizados, na tentativa de compreender a individualidade dos acometimentos e a pluralidade dos comportamentos diante da patologia para, dessa forma, gerar estratégias de acolhimento à saúde, sensíveis às particularidades, porém eficientes na manutenção do autocuidado, sobretudo, para com os indivíduos mais vulneráveis, os quais convivem com a persistência do preconceito e dos "tabus" alicerçados pela ausência de esclarecimentos com uma linguagem acessível, incentivadora e de políticas públicas capazes de transformar o cenário da hanseníase no país, que há tempos é negligenciado pelo sistema público de saúde. 


\section{Referências}

BARBOSA, J. C. et al. Atenção pós-alta em hanseníase no Sistema Único de Saúde: aspectos relativos ao acesso na região Nordeste. Cadernos Saúde Coletiva. v. 22, n. 4, p. 351-358, 2014.

BATISTA, T. V. G.; VIEIRA, C. S. C. A.; DE PAULA, M. A. B. A imagem corporal nas ações educativas em autocuidado para pessoas que tiveram hanseníase. Physis - Rev. Saúde Coletiva. v. 24, n. 1, p. 89-104, 2014.

BELUCI, M.; BORGATO, M. H.; GALAN, N. G. A. Avaliação de cursos multiprofissionais em hanseníase. Hansenologia Internationalis (Online). v. 37, n. 2, p. 47-53, 2012.

BRASIL. Ministério da Saúde. Hanseníase: o que é, causas, sinais e sintomas, tratamento, diagnóstico e prevenção. Disponível em: https://www.saude.gov.br/saude-de-a-z/hanseniase. Acesso em: 16 de fev. de 2020.

BRASIL. Ministério da Saúde. Secretaria de Vigilância em Saúde. Departamento de Vigilância Epidemiológica. Guia de apoio para grupos de autocuidado em hanseníase. Brasília: Ministério da Saúde, 2010.

BRASIL. Ministério da Saúde. Secretaria de Vigilância em Saúde. Departamento de Vigilância das Doenças Transmissíveis. Guia prático sobre a hanseníase. Brasília: Ministério da Saúde, 2017.

CASTRO, R. N. C. et al. Avaliação do grau de incapacidade física de pacientes com hanseníase submetidos ao Dermatology Quality Life Index em centro de referência e unidades básicas de saúde de São Luis, MA. Rev. Bras. Clin. Med. v. 7, p. 390-392, 2009.

CORRÊA, B. J. et al. Associação entre sintomas depressivos, trabalho e grau de incapacidade na hanseníase. Revista Acta Fisiátrica. v. 21, n. 1, p. 1-5, 2014.

D'AZEVEDO, S. S. P. et al. Percepção de pacientes com dois grupos de autocuidado. Rev. Enferm. v. 12, n. 6, p.1633-1639, 2018.

DOURADO, G. O. L. et al. Association between alcoholic beverages consumption and sociodemographic conditions in people with leprosy. Bioscience Journal. v. 35, n. 3, 2019.

GALAN, N. G. A. et al. Avaliação da prática do autocuidado domiciliar em hanseníase. Hansen Int. v. 39, n. 2, p. 37-45, 2016.

GONÇALVES, N. V. et al. A hanseníase em um distrito administrativo de Belém, estado do Pará, Brasil: relações entre território, socioeconomia e política pública em saúde, 2007-2013. Rev. Pan-Amaz. Saúde. v. 9, n. 2, p. 21-30. 2018 
LANZA, F. M. et al. Perfil epidemiológico da hanseníase no município de Divinópolis, Minas Gerais. Rev. Enferm. UFSM. v. 2, n. 2, p. 365-374, 2012.

LAURINDO, C. R. et al. Acesso à orientação quanto ao autocuidado por pessoas diagnosticadas com hanseníase em um município da Zona da Mata Mineira. HU Revista. v. 44, n. 3, p. 295-301, 2018.

LIMA, M. C. V. et al. Práticas de autocuidado em hanseníase: face, mãos e pés. Rev. Gaúcha Enferm. v. 39, p. 1-7, 2018.

MAIA, F. B. et al. The use of assistive technology to promote care of the self and social inclusion in patients with sequels of leprosy. PLoS Negl Trop Dis. v. 10, n. 4, p. e0004644, 2016.

PINHEIRO, M. G. C. et al. Conhecimento sobre prevenção de incapacidades em um grupo de autocuidado em hanseníase. Revista Mineira de Enfermagem. v. 18, n. 4, p. 895-906, 2014.

REIS, A. S. et al. Sobreposição de casos novos de hanseníase em redes de convívio domiciliar em dois municípios do Norte e Nordeste do Brasil, 20012014. Cad. Saúde Pública. v. 35, n. 10, p.1-13, 2019.

RIBEIRO, M. D. A.; SILVA, J. C. A.; OLIVEIRA, S. B. Estudo epidemiológico da hanseníase no Brasil: reflexão sobre as metas de eliminação. Rev. Panam. Salud Publica. v. 42, p. e42, 2018.

RODINI, F. C. B. et al. Prevenção de incapacidade na hanseníase com apoio em um manual de autocuidado para pacientes. Fisioter. Pesqui. v. 17, n. 2, p 66-157, 2010.

ROLIM, M. F. N. et al. Fatores relacionados ao abandono ou interrupção do tratamento da hanseníase. Journal of Medicine and Health Promotion. v. 1, n. 3, p. 254-266, 2016.

SANTOS, A. K. C.; ARAÚJO, T. A.; OLIVEIRA, F. S. Farmacoterapia e cuidados farmacêuticos da gripe e resfriado. Journal of Biology \& Pharmacy and Agricultural Management. v. 16, n. 2, p.137-155, 2020.

SILVA, K. R.; LOPES W. M. P. S.; COSTA J. J. Perfil clínico e epidemiológico de reações hansênicas. Rev. Enferm. UFPI [Internet]. v. 8, n. 3, p. 48-54, 2019.

SILVA, M. C. D.; PAZ, E. P. A. Educação em saúde no programa de controle da hanseníase: a vivência da equipe multiprofissional. Escola Anna Nery. v. 14, n. 2, p. 223-229, 2010.

SOARES, R. X. et al. Dor em neonatos: avaliações e intervenções farmacológicas e não farmacológicas. Revista de Ciências Médicas e Biológicas. v. 18, n. 1, p. 128-134, 2019. 
SOUSA, A. A. et al. Adesão ao tratamento da hanseníase por pacientes acompanhados em unidades básicas de saúde de Imperatriz - MA. SANARE Revista de Políticas Públicas. v. 12, n. 1, p. 6-12, 2013.

SOUZA, I. A. et al. Autocuidado na percepção de pessoas com hanseníase sob a ótica da complexidade. Esc. Anna Nery. v. 18, n. 3, p. 510-514, 2014.

SOUZA, M. T.; SILVA, M. D.; CARVALHO, R. Revisão integrativa: o que é e como fazer. Einstein. v. 8, n. 1, p. 102-106, 2010.

\section{$6 \quad$ Agradecimentos}

À Liga Acadêmica de AnatoHistoFisiologia da Universidade do Estado do Pará, por proporcionar aos seus ligantes o incentivo à pesquisa científica. 